University of Nebraska - Lincoln

DigitalCommons@University of Nebraska - Lincoln

June 1982

\title{
Biological Effects of Low Doses of lonizing Radiations: Particle Tracks in Radiobiology
}

Robert Katz

University of Nebraska-Lincoln, rkatz2@unl.edu

Werner Hofmann

University of Salzburg, Austria

Follow this and additional works at: https://digitalcommons.unl.edu/physicskatz

Part of the Physics Commons

Katz, Robert and Hofmann, Werner, "Biological Effects of Low Doses of lonizing Radiations: Particle Tracks in Radiobiology" (1982). Robert Katz Publications. 111.

https://digitalcommons.unl.edu/physicskatz/111

This Article is brought to you for free and open access by the Research Papers in Physics and Astronomy at DigitalCommons@University of Nebraska - Lincoln. It has been accepted for inclusion in Robert Katz Publications by an authorized administrator of DigitalCommons@University of Nebraska - Lincoln. 
Published in Nuclear Instruments and Methods 203 (1982), pp. 433-442.

Copyright (C) 1982 North-Holland Publishing Company/Elsevier. Used by permission.

http://www.sciencedirect.com/science/journal/01689002

Research for this article was supported by the U.S. Department of Energy and the Max Kade Foundation.

Submitted March 26, 1981; revised February 23, 1982.

\title{
Biological Effects of Low Doses of Ionizing Radiations: Particle Tracks in Radiobiology
}

\author{
Robert Katz \\ University of Nebraska-Lincoln, Lincoln NE 68588-0111, U.S.A. \\ Werner Hofmann \\ Division of Biophysics, University of Salzburg, Erzabt-Klotz-Str. 11, A-5020 Salzburg, Austria
}

\begin{abstract}
A radiation field is made up of a tangle of particle tracks, from the primary particle and secondary and higher generation electron interactions, well isolated at low doses and with multiple intersections in cell nuclei at high doses. Low dose effects in multicellular systems are therefore the sum of individual track structures. Until we can state with confidence the structure of a particle track in biological matter for all end-points of interest, at least as well as we can for nuclear emulsions, our knowledge of low dose effects should be regarded as uncertain and inadequate. In this context "track structure" means the response of physical and biological systems along the path of the particle, and depends on the observed end-point as well as on the identity of the particle. For mammalian cell killing and a few other biological end-points, track theory and experimental radiosensitivity parameters allow us to construct schematic models. If we take a particle track to consist of a sequence of inactivated cells strung along the path of a particle, neither electrons nor protons leave a track in a compact mammalian cell structure. At most there is an occasional killed cell at the end of the range of a proton or an electron where the particle stops in the nucleus of a cell, with probability less than 0.3 for a proton, and less than 0.01 for an electron. The variety of potential targets whose size may be compared to the measured inactivation cross-section and the lack of a fully consistent theory of RBE make it impossible to decide, from this information alone, whether cell killing is an individual (1-hit) or cooperative (many-hit) phenomenon, especially for electrons. A similar analysis of epidemiological data for cancer induction leads to probabilities and action cross-sections so low as to make a linear extrapolation implausible. In assigning quality factors at highest LET values we should consider that heavy ions inactivate cells, so that neither mutation nor transformation can represent a hazard. At low doses, when only isolated inactivated cells are produced whose function may be restored by repopulation, it is difficult to see why high LET radiations are assigned the highest quality factors.
\end{abstract}

\section{Introduction}

The term "particle track" is commonly used in physics and in radiation biology in a variety of ways. Unless otherwise stated we will here use the words "particle track" to relate to the pattern of effect generated in the physical or biological detector by the passage of the primary particle and its associated secondary and higher generation $\delta$-rays, such as the pattern of developed grains in nuclear emulsions or of inactivated cells in biological matter. We must be aware that the structure of the track depends on the end-point being observed. In a photographic emulsion the pattern of developed grains can be altered, both qualitatively and quantitatively by changes in developer composition, dilution, temperature, and time. We must expect that the track structure in emulsion would also be different if the observation was made with an ultra-violet microscope, for the size of a developed grain in relation to the wavelength of light also affects our perception of the "end-point." The words "track," "detector," and "end-point" are intended to encompass all of these questions.

In most cases of radiation exposure of the general public, or of occupational exposure of the individual, man is exposed to low levels of ionizing radiations. Despite the importance of low dose effects, no exact definition exists of what is meant by "low." Typically low doses can be expected in environmental natural background exposure. In the context of this paper we speak of low doses when biological targets, such as cells or cell organelles, are traversed only by single charged particles and their associated $\delta$-rays, and where intertrack interactions can be neglected. Although man is exposed to low dose levels during his entire lifetime, only rough and controversial information can be derived from epidemiological investigations, the main problem being the synergistic interference of other environmental factors, e.g. co-carcinogens. Their interference can be minimized 
at higher radiation doses. Thus we may take uranium miner data as representative of the human reaction to radon decay products, or we may seek to improve the statistical accuracy of in-vitro cellular experiments, or of in-vivo animal experiments by high dose exposures. In general radiobiological experiments at high doses are reasonably well understood. The problem of radiation effects at low doses is therefore mainly the problem of extrapolation from high to low doses and must be based on theoretical assumptions. Depending on the theoretical model used, different results are obtained at low doses [1]. It is therefore of utmost importance that the model used for extrapolation be testable at low doses with other detectors than biological systems, but whose response to radiation can be thought to parallel biological response in some significant way.

Experiment alone cannot provide an evaluation between dose and effect at levels of risk between $10^{-5}$ and $10^{-7} \mathrm{~Gy}^{-1}$ needed for estimating radiation hazards to large populations [2].

In first approximation such effects arise from the interaction of isolated energetic particles with biological targets. The low dose problem thus reduces to one of understanding the structure of a particle track in biological matter for the required variety of end-points. Until this can be accomplished our knowledge of the biological effects of low doses of ionizing radiations must be regarded as incomplete and speculative. Our knowledge of the structure of particle tracks, of low dose effects, must come from an interpretation of high dose effects. For this reason cell killing is the main biological end-point considered in the present context.

We have achieved a detailed model of cell killing by high LET radiations of demonstrated predictive value, and from which it is possible to extract a description of the structure of individual particle tracks (for this endpoint) from cell survival experiments at high doses with high LET radiations, such as beams of energetic heavy particles. However, mutagenesis and transformation toward malignancy are also discussed here, for these represent the most important genetic and somatic hazards to man, though the experimental data are much more limited.

Selective stains which reveal the tracks of charged particles for end-points of interest are either only partially available or fail to ascertain that the effects are produced by single particles. To develop a picture of particle tracks in biology we must rely on a theory of biological response to radiations of different quality which is so constructed as to parallel a theory of particle tracks in nuclear emulsions, and which has been tested there and in a variety of other detectors. If the theory reasonably describes bulk effects in emulsions and other detectors, and particle tracks in emulsions, while also describing bulk effects in biology, we may have some confidence in its extrapolation to particle tracks in biol- ogy, and hence to the effects of low levels of radiation. Neither the theories which describe biological effects nor those which describe particle tracks in emulsion can be considered exact. Nevertheless they form a logical and computational structure which describes patterns of response of both biological and physical detectors to arbitrary radiation fields.

\section{The 1-or-more hit detector}

One-or-more hit detectors are characterized as having an exponential response to dose and a decline in response with an increase in the "LET" of the incident radiation.

The LET or "linear energy transfer" refers to a variety of restricted energy loss, or stopping power [3]. In the present context we imply no restriction and use the term LET to imply LET $_{\infty}$, the total rate of energy loss.

To describe the properties of a 1- (or-more) hit detector theory requires knowledge of the following detector parameters: $E_{0}$, the dose of gamma-rays at which there is an average of 1-hit per target, $\alpha_{0}$, the radius of the target (or $\kappa=E_{0} \alpha_{0}^{2} / 2 \times 10^{-2} \mathrm{~J}^{-1} \mathrm{~m}$ ), and $N_{0}$, the number density of targets, as well as the fact that the detector responds exponentially to radiations, in the form of the 1-or-more hit cumulative Poisson distribution. Together with the parameters of the ionizing particle, the radial distribution of the average local dose about the path of an ion, $\bar{E}\left(z^{*}, \beta, t, \alpha_{0}\right)$, is needed. Here $z^{*}$ is the effective charge number of the ion, $\beta$ is its speed relative to the speed of light, and $t$ is the radial distance from the ion's path to the center of the sensitive target. This distribution can be determined, either from measurement or by calculation from the properties of the medium, knowledge of $\delta$-ray production, and of the subsequent electron interactions by which the energy lost by the heavy ion is transferred to the medium.

Neither the parameters of the detector nor the radial distribution of dose are known precisely. Nevertheless the relationships are such that with some adjustment of parameters the overall form of the detector response to radiations of different "LET" and the structure of a particle track can be well fitted and even predicted by the theory.

Typically the point distribution in local dose is given by an expression of the form

$$
E(t)=C_{1} z^{* 2} / \beta^{2} t^{2}
$$

from a radial distance $t \simeq 15 \mathrm{~nm}$ to a maximal range $t_{\max }$ which lies in the neighborhood of

$$
t_{\max }=C_{2} T^{5 / 3},
$$

for a particle with kinetic energy per unit mass of value $T$.

The only available experimental data $[4,5]$ yield val- 
ues of $C_{1}$, in water, in the neighborhood of $150 \mathrm{~Gy} \mathrm{~nm}{ }^{2}$, within $25 \%$ fluctuation, while calculations $[5,6,8]$ yield similar values. The constant $C_{2}$ has the value $0.25 \mu \mathrm{m}$ $(\mathrm{MeV} / \mathrm{amu})^{-5 / 3}$, for $t_{\max }$ is in $\mu \mathrm{m}$ when $T$ is in $\mathrm{MeV} / \mathrm{amu}$. The average local dose $\bar{E}$ can then be obtained by averaging the point dose $E$ over the sensitive site of radius $\alpha_{0}$ centered at a radial distance $t$ from the ion's path.

The theory of the 1-hit detector describes the behavior of normally developed sensitive nuclear emulsions very well [9]. In essence we find the radial distribution in effect about an ion's path from the radial distribution in local dose deposited by delta electrons [equation (l)] and the response of the detector to gamma-rays. For a uniform irradiation by gamma-rays with dose $D$, the dose-response relationship for a 1-hit detector is described by

$$
P(D)=1-\mathrm{e}^{-D / E_{0}} .
$$

Substituting $\bar{E}$ for $D$, then enables us to calculate the linear density of developed grains, the track width or opacity, and to prepare computer models of particle tracks whose appearance compares well with microphotographs of real tracks [10-13]. The radiosensitivity parameters of the emulsion are partly obtainable from the manufacturer $\left(\alpha_{0}\right.$ and $\left.N_{0}\right)$, while $E_{0}$ is sometimes measured from sensitometry after gamma-irradiation, and is sometimes fitted to the appearance of a particle track.

The theory of the 1-hit detector also provides a very good description of the variation of the inactivation cross-sections of dry enzymes and viruses bombarded by heavy ion beams of different "LET." Here the cross section $\sigma$ is identified with the radially integrated probability $P$ of inactivation of the targets, the enzyme or virus molecules, by the passage of a single ion:

$$
\sigma=\int_{0}^{t_{\max }} 2 \pi t P \mathrm{~d} t
$$

The decline in the yield of single strand break events in $\Phi x-174$ with an increase in "LET" [14] suggests that this is also a 1-hit process. Indeed single strand break events are defined by the authors as l-or-more breaks in a strand of the DNA double helix, as consistent with a l-or-more hit process. We have been able to fit these strand break data with the assumption that this process is described by the parameters $E_{0}=7 \mathrm{kGy}$ and $\alpha_{0}=10$ $\mathrm{nm}$, not far from the parameters used in describing the inactivation of this virus [8]. To reasonable approximation radical production is also a 1-hit process, often of biological interest.

Particle tracks in these biological 1-hit detectors, with inactivation as the biological end-point, will have similar appearance to particle tracks in 1-hit emulsions, except for questions of scale. Indeed, exactly the same procedure used for emulsions [12] may be followed to yield the tracks in these biological detectors. The tracks will exhibit a grain-count regime, where the inactivated targets are openly strung along the ion's path like beads on a string, when the probability $P$ for inactivating targets traversed by the ion is small. In this case the inactivation cross-section is also small when compared to the geometric cross-section. With increasing "LET," as for a heavier or a slower ion, the inactivated targets are strung more closely together to present an increasingly filled "core" and a developing "track-width," as the inactivation cross-section exceeds the geometrical crosssection of the target. Experiments with virus inactivation have found inactivation cross-sections more than an order of magnitude greater than the geometric crosssection, while with emulsions the track width has been observed to be as much as 100 times the diameter of the undeveloped emulsion grain.

In general, the tracks formed by charged particles in 1-hit detectors are reasonably well understood. For such detectors we can claim to understand the effects of low doses of ionizing radiations. For 1-hit detectors the linear extrapolation to low dose is precisely correct.

\section{Biological cells}

Our understanding of the structure of particle tracks in tissue, or in a matrix of biological cells in-vitro, poses a much more severe problem, different for every different end-point. We do not truly know the size nor the location of the sensitive targets, nor the number of these targets which must be hit to yield a particular end-point, aside from saying that they are in the nucleus or that they may be associated with the DNA or with the nuclear membrane [15]. Repair processes affect biological response, but we have no quantitative understanding of the effects of radiation on these processes. We cannot be certain as to the shape of survival curves or of other dose-effect relationships, especially at low doses. Most analyses take the form of statistical curve fitting to high dose data, but these suffer from the inherent problem that the statistical models assume a homogeneous population, while experimental populations are heterogeneous [15]. It is currently popular (widely accepted) to use a linear-quadratic model, but such a model is by no means uniquely fitted to the available data.

It has been argued that there is an intimate relationship between the shape of the survival curve after gamma-irradiation (low "LET") and the "RBE" after high "LET" irradiations [17]. According to the ICRU [3], the Relative Biological Effectiveness (RBE) is defined as the ratio of the absorbed dose of a reference radiation to the absorbed dose of a test radiation to produce the same level of biological effect, other things being equal. The relationship between low and high LET radiations is based on the assumption inherent to track theory that the difference between low and high "LET" radiations 
lies in (1) the temporal scale of the irradiation and (2) the microscopic concentration of local dose about the path of a heavy ion, and that sensitive targets respond to the dose of secondary electrons, irrespective of their origin. To the extent that temporal differences may be neglected, it follows that the probability for cell killing relative to the absorbed dose after gamma irradiation must be supralinear if an RBE greater than 1 is to be observed for any high "LET" radiation. For a more extensive discussion of these problems the reader is referred to references 15, 17-20.

When considering this problem from a theoretical viewpoint, it is imperative that we ask what the implications of a low "LET" survival curve are for the structure of particle tracks. These, after all, contain a high dose region close to the particle's path where dose rate effects can be of consequence, and a low dose region far from the path where the question of dose rate is meaningless, because it is unlikely that two electrons from the same ion pass through the same target or even the same cell nucleus. In the periphery of an ion's path the only difference between delta-rays from a heavy ion and isolated Compton electrons from a photon irradiation lies in their energy spectra. The question of extrapolation of low doses of low LET radiations cannot be separated from the question of the RBE of high LET radiations.

Physical detectors more complex than 1-hit detectors have been treated as $C$-or-more hit systems, with four parameters, the hittedness $C, E_{0}, \alpha_{0}$ (or $\kappa$ ) and $N_{0}$. Application of the theory to biological cells whose survival curve after gamma-irradiation is represented by the multi-target single-hit statistical model, requires an additional parameter, the cross sectional area of the nucleus of the cell, for the sensitive sites are distributed throughout the nucleus, perhaps like "beans" in a "bean-bag." These parameters are then $m$, the extrapolation number of the gamma-ray survival curve, or the number of (1-hit) sensitive subcellular sites which must be inactivated to inactivate the cell, $E_{0}$, the dose at which there is an average of 1-hit per target, $\kappa$, the value of $z^{* 2} / 4 \beta^{2}$ at which the plateau value of the cellular inactivation cross-section is achieved, $\sigma_{0}$, the plateau value of the extrapolated cross-section (which approximates the geometrical cross-section of the cell nucleus), and $N_{0}$, the number density of cells, needed for track simulation, but not for survival curves. Thus for biological cells there are four fitted parameters from which the formulas of the theory must yield survival curves for an arbitrary radiation field for which we know the particle-energy spectrum $[7,21]$. The parameters are typically fitted to survival data for particles used in bombardments at energies less than $10 \mathrm{MeV} / \mathrm{amu}$, and are then used for calculations for all radiation fields.

The theory has been tested for neutron and pion irradiations [22], and irradiations with admixtures of neu- trons and gamma-rays [23]. Most recently, in connection with a relatively primitive model of the secondary fragment production in heavy ion beams, it has been used to predict the inactivation of T-1 cells as a function of depth in energetic $\mathrm{C}, \mathrm{He}$, and Ar beams [24]. Indeed calculations from in-vitro parameters have been shown to yield in-vivo values of the "RBE" vs. neutron dose for several different biological end-points [25], suggesting that the parameters of the model and the calculations made from it are valid for extrapolation to the structure of particle tracks in both in-vitro and in-vivo systems. In sum then, track structure theory in its extension to the "RBE" of high "LET" radiations has predictive value in spite of a not completely resolved conflict $[17,19]$ between experiment and the demands of the theory in regard to the initial slope of the survival curve after gamma-irradiation. The success of the theory with heavy ion irradiations [24], and with mixed radiation environments [22,23] does account for the effects of particle track structure in high "LET" irradiations and therefore lends itself to a calculation of the structure of the tracks of single particles in biological matter.

\section{Particle tracks in biological matter}

According to track theory, an ion of atomic number $Z$, effective charge number $z^{*}$, given by

$$
z^{*}=Z\left[1-\mathrm{e}^{-125 \beta Z^{-2 / 3}}\right],
$$

when moving at speed $\beta$ relative to the speed of light, has probability $P$ for inactivating a cell whose nucleus it threads, given by

$$
P=\left[1-\mathrm{e}^{-z^{* 2} / \kappa \beta^{2}}\right]^{m} .
$$

For T-1 cells, aerobically irradiated, $\kappa=1000$ and $m=$ 2.5. Equation (6) describes the inactivation probability in the "grain-count" regime, where inactivated cells lie along the ion's path, like beads on a string. Interested only in relatively low $Z$ particles, we can here neglect the "track-width" regime. With the aid of equation (6), knowledge of the radiosensitivity parameters $\kappa, m[7,21]$ and $N_{0}$, and a table of residual range $R$ vs. $\beta$ in water, we are able to construct a picture of the track of a heavy ion in biological matter with inactivation as the end-point.

To simulate a track we divide the path of the ion into range intervals $l_{i}$, at $\beta=0,0.01,0.02, \ldots$ and find $P_{i}$ from $\beta_{i}$ at the high energy end-point of each interval. If the average distance between the centers of the cells is $\lambda$, then the average number of cells in the $i$ th interval is $l_{i} /$ $\lambda$, and the average number of cells killed in the $i$ th interval is $P_{i} l_{i} / \lambda$. These cells are placed at random in the $i$ th interval. 
Particle Tracks in "Close Packed" T-1 Human Kidney Cells (Aerobic) in $1 \mathrm{~mm}$ segments
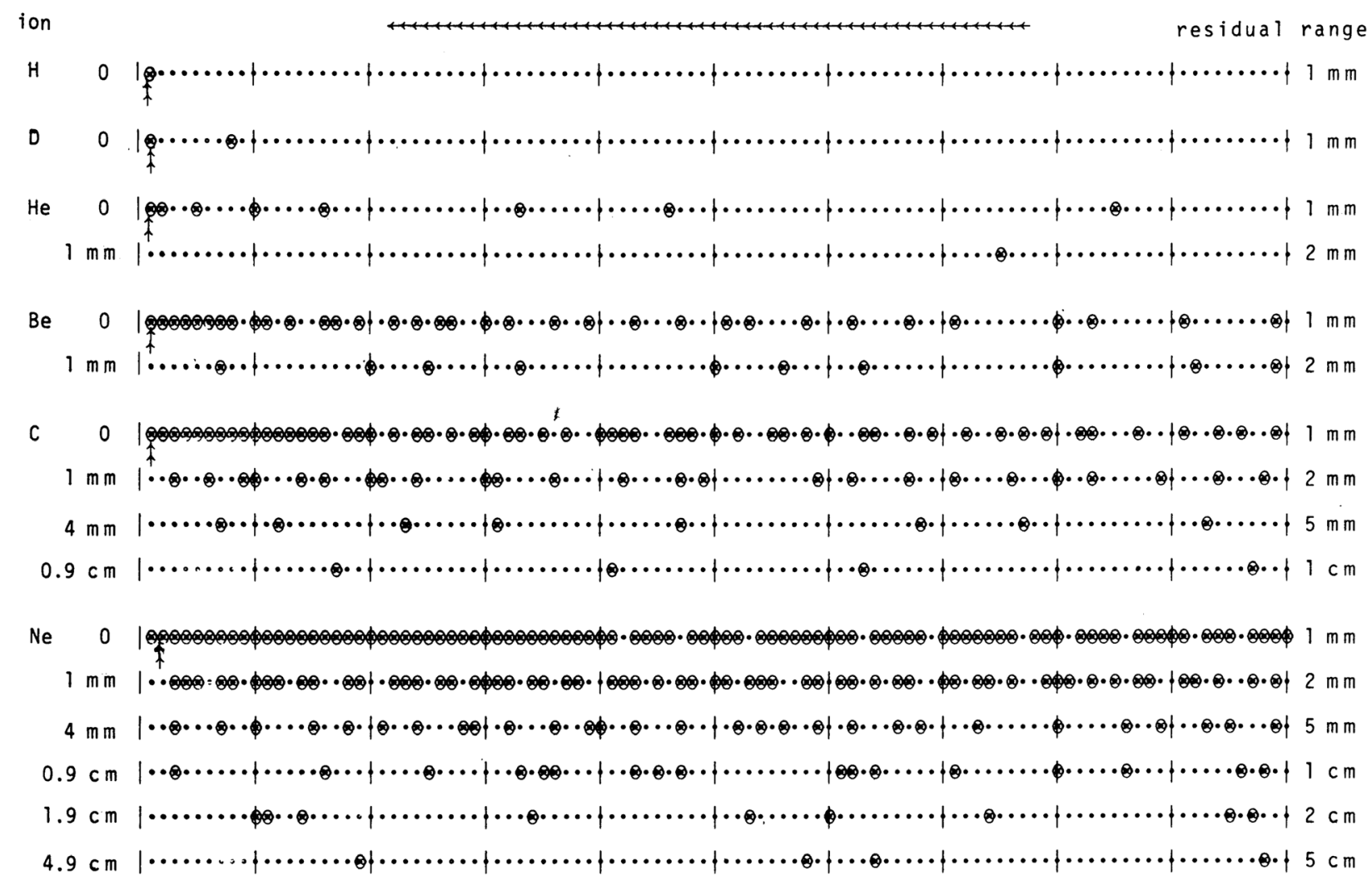
$\ddagger$ indicates position of Bragg peak.
Each cell is taken to have a diameter of 10 microns, with the leftmost end of the first cell at the residual range indicated at left. Cells are close-packed and aligned along the ion's path.
$\circledast$ indicates a cell killed by a heavy ion moving from right to left, in the "ion-kill mode."
- indicates a cell whose nucleus has been intersected by the moving heavy ion and which experiences only sub-lethal damage.

Figure 1. Schematic simulation of the tracks of heavy ions in an "emulsion" of aligned, close-packed cells (at $10 \mu \mathrm{m}$ intervals) having the radiosensitivity parameters of aerobically irradiated T-1 kidney cells.

Figure 1 shows particle tracks constructed for cells having the radiosensitivity parameters of aerobically irradiated T-1 kidney cells. These are assumed to form a close packed structure with $N_{0}=10^{6}$ cells $/ \mathrm{mm}^{3}$ and with cell spacing $\lambda=10 \mu \mathrm{m}$. Track segments $1 \mathrm{~mm}$ long are shown, at selected ranges, slowing down from right to left, for ions from protons to argon. 


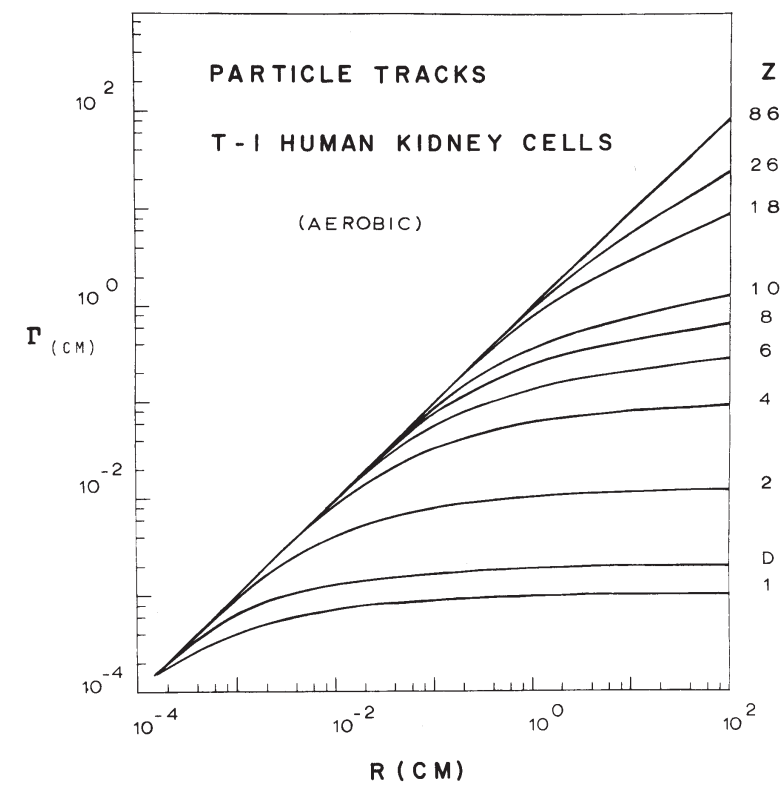

Figure 2. $\Gamma\left(=\int P d R\right)$ in $\mathrm{cm}$, vs. range $T$, in $\mathrm{cm}$, for different ions in a cellular matrix. The average number of cells killed by an ion of residual range $R$ is found by dividing $\Gamma$ by the average distance between intersections, $\lambda$. The fraction of intersected cells killed by an ion is $\Gamma / R$.

A second representation of these data is given in Figure 2, displayed in a different manner. Using the same formulation we calculate $\Gamma=\sum P_{i} l_{i}$ and $R=\sum l_{i}$. Since $\Gamma /$ $R$ is the fraction of intersected cells inactivated by a particle of range $R$, the $45^{\circ}$ line represents residual range values at which each traversed cell will be killed. Multiplying $\Gamma / R$ by the total number of cells along the particle range, $R / \lambda$, the number of cells inactivated by a particle of residual range $R$ will be $\Gamma / \lambda$.

We must issue a cautionary note about the quality of the simulation at the very end of the track, where the range of delta-rays may be too small to bathe even several of the sensitive elements of a cell, let alone an entire cell nucleus, as may be seen from equation (2). A similar cautionary note deals with cells whose nuclei are barely grazed by the ion's path, but which may be inactivated by its delta-rays, as we see from equation (1). Taking $C_{1}$

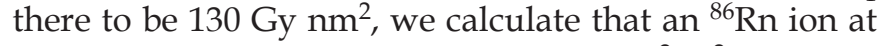
$\beta=0.15$, with $T=10.66 \mathrm{MeV} / \mathrm{amu}$, has $z^{* 2} / \beta^{2}=1.255 \times$ $10^{5}$. This leads to $t_{\max }=12.6 \mu \mathrm{m}$, and to a local dose of $1.8 \mathrm{~Gy}$ at $t=3 \mu \mathrm{m}$. The existence of a predicted cellular track-width regime has recently been verified with bacterial spores [27] and yeast cells [28].

Experiments by Goodhead et al. [29] yield the estimate that helium ions at $90 \mathrm{keV} / \mu \mathrm{m}$ produce 0.03 to 0.06 lethal lesions $/ \mu \mathrm{m}$ through nuclei $7 \mu \mathrm{m}$ thick in mammalian cells. This finding is in reasonable agreement with our calculation for T-1 kidney cells, where we find $P=$ 0.36 at $\beta=0.06$, where the stopping power is $77 \mathrm{keV} / \mu \mathrm{m}$.
Experiments by Datta et al. [30] with ${ }^{241} \mathrm{Am}$ alpha particles find that the greatest inactivation probability for helium ions which stop in the nucleus of a cell is 0.43 , and that this probability drops both for lower and for higher energies. For comparison our estimate for a helium ion having a range of $8 \mu \mathrm{m}$ is 0.8 . Experiments by Lloyd et al. [31] give similar results. There are some interpretive problems associated with the determination of the crosssectional area of a plated nucleus, estimated by different investigators as from 40 to $300 \mu \mathrm{m}^{2}$, leading to discrepancies in the estimated probability for killing by an alpha particle. Thus there are problems which remain unsolved in both calculation and measurement. Within this grey area, however, calculation and experimental results agree, even for stopping helium ions. Contrary to the situation with alpha particles, there are unfortunately no comparable experimental data for protons. In Figure 1 we have represented the last cell whose nucleus is intersected by a proton as killed. This is an overestimate, for our calculations indicate that the probability of killing the last intersected cell is about $1 / 3$. Once again, at the very end of the track these calculations probably overestimate cell killing because of the limited range of the delta rays. For example, $t_{\max }=0.23 \mu \mathrm{m}$ at the residual proton range of $6.7 \mu \mathrm{m}$. But the diameter of the cell nucleus may be $8 \mu \mathrm{m}$.

The tracks of heavier ions, with cell killing as the endpoint present no particular problems, for all intersected cells are killed at the very end of the track, where the greatest uncertainties exist in the calculation. We will return to this point in our discussion of cancer induction. Since cancer cannot be induced in killed cells, a question must be raised about the high values assigned to the quality factor at the highest "LET."

\section{Electron tracks}

The evaluation of track structure for electrons in biological systems poses a difficult problem. The fundamental question is: Can a single electron kill a cell? It is not enough to say that at track-end electrons are high "LET" particles. As Lloyd et al. [31] have pointed out, we must consider the probability per unit path length. And "high LET electrons" have a very limited residual range.

Track theory starts from the response of a bulk medium to a dose of low "LET" radiation (gamma-rays), a tangle of electron tracks, and maps this response about the path of a heavy ion. It therefore does not speak directly to the question of the formation of electron tracks. To do so requires a theory of electron action cross-sections and a connection between these cross-sections and a macroscopic dose-effect relationship for a bulk medium. This is a task for future research. 
In the framework of the present theory we can estimate the effect of an electron on a biological system by calculating the probability for cell killing for a "pseudoelectron," a particle having the charge and mass of an electron which behaves in accordance with expressions derived for and tested with heavy ions. Applying equation (6) to this problem, and neglecting branching secondary electrons of sufficient energy to form tracks of their own, we conclude that $\Gamma=2.3 \times 10^{-2} \mu \mathrm{m}$ for a 1 $\mathrm{MeV}$ pseudo-electron (range in water about $4 \mathrm{~mm}$ ) and $2.2 \times 10^{-2} \mu \mathrm{m}$ for a $15 \mathrm{keV}$ pseudo-electron. It is only at track end that such a particle has even a slight chance of killing a cell. However, when an electron moves slowly enough that the probability of inactivating a cell is large, its residual range is very small. Taking the mean spacing between cells to be $10 \mu \mathrm{m}$, this particle can be expected to kill $2.2 \times 10^{-3}$ cells in a close-packed medium, at 15 $\mathrm{keV}$ and $2.3 \times 10^{-3}$ cells at $1 \mathrm{MeV}$ initial energy, neglecting the production of energetic secondary electrons.

These results are consistent with experimental findings in microbeam irradiations with electrons of $15 \mathrm{keV}$ initial energy, by Datta et al. [30] who found that about 1,000 electrons incident on the cell over the area of the nucleus were required to kill a cell. Because of scattering, only about half of these electrons were estimated to have penetrated the nuclear membrane. This leads to an estimate that the maximum probability for cell killing by track-end electrons is $2 \times 10^{-3}$, for $15 \mathrm{keV}$ electrons were more effective than incident electrons of either higher or lower energies in these experiments. As always in any high fluence experiment, we cannot be certain that the effect is produced from a single incident electron (ionkill) rather than the cumulative effect of several incident electrons (gamma-kill). Nor should we infer that a single electron action is involved from the apparent coincidence between action cross-sections and the physical size of a possible target, as has been suggested by Cole et al. [32], since action cross-sections may be an order of magnitude greater or smaller than the actual cross-sectional area of the target $[7,8]$. Nevertheless the upper limit established by experiment for the probability that a $15 \mathrm{keV}$ electron inactivates cells agrees with the calculation for "pseudo-electrons."

Another way of approaching the problem is through the measured initial slope of survival curves after electron or gamma-ray irradiation. If an electron of initial energy $w$ and all its secondaries kills $v$ cells when fully absorbed in a matrix containing $N_{0}$ cells per unit volume in a medium of unit density, the initial slope $\alpha$ of the dose-effect survival curve is given as

$$
\alpha=v / N_{0} w \text {. }
$$

This result is obtained by noting that an irradiation with a fluence of $F$ electrons per unit mass, of initial energy $w$ deposits a dose $D=F w$, while if each electron and all its sec- ondaries kills $v$ cells, the fraction of cells killed is $F v / N_{0}$. Inserting these quantities into the usual expression for the initial slope of the survival curve $N / N_{0}=1-\alpha D+\ldots$. leads to equation (7).

Typical in-vitro experiments with mammalian cells contain a maximum concentration of $10^{5}$ cells $/ \mathrm{ml}$ [33]. Then, if $1 \mathrm{MeV}$ electrons produce a survival curve with initial slope $\alpha=10^{-5} \mathrm{~Gy}^{-1}$ derived from gamma-irradiation of V-79 cells [34], we find $v=1.6 \times 10^{-6}$ cells killed per incident electron and all its secondaries. In these experiments much energy is deposited in the culture medium. To evaluate the effect of a single electron in a cell culture from which the medium has been extracted, or for in-vivo tissue experiments, we suppose that $N_{0}=10^{9}$ cells $/ \mathrm{ml}$, leading to a value of $v$ that is $10^{4}$ times greater than before. Then a $1 \mathrm{MeV}$ electron and all its secondaries might kill $1.6 \times 10^{-2}$ cells when $\alpha=10^{-5} \mathrm{~Gy}^{-1}$.

We must conclude that an electron does not make an observable track in tissue, even for a survival curve whose initial slope is 10 times greater than that for which the calculation is made. At most an occasional single cell is inactivated at path end in such a way that it would be impossible to identify observationally with single electrons.

\section{Other biological end-points}

Contrary to cell killing we do not have radiosensitivity parameters for other end-points, such as DNA double strand breaks, G-2 block, chromosome breaks, transformation, or mutation. At best we can estimate response relative to cell killing from data like that presented by Cole et al. [32], which indicates that transformation and mutation are about $1 / 400$ and $1 / 40000$, respectively, as likely as lethality from a single alpha particle. On the other hand double strand breaks are about 10 times more likely than lethality, while G-2 block and chromosome breaks only about 3 times. For $1 \mathrm{MeV}$ electrons and all their progeny we can estimate that 1 electron in 24,000 would yield a transformed cell, while one electron in 2,400,000 would yield a mutation.

For such rare events the problem arises that "track" loses its proper meaning as an observable entity. A particle track can also be regarded as the probability distribution for a defined end-point along an ion's path, which becomes observable only if the probability exceeds a certain level. For very low probabilities a specified biological effect in a single cell is likely to arise by the cooperative action of two or more electrons passing through the nucleus of the cell when this leads to a joint probability of appropriate value.

From all the different biological end-points, cancer induction represents the most important somatic hazard in radiation protection. We thus wish to explore the 
question: What is the probability for a $1 \mathrm{MeV}$ electron to cause a cancer? Since transformation toward malignancy is regarded as the initial event in carcinogenesis, in-vitro transformation experiments can supply part of the needed information. Thus we will get an upper limit for the probability for cancer induction, because not every transformed cell will develop a tumor. From these data we can derive a probability for transforming a cell by a single $1 \mathrm{MeV}$ electron of between $10^{-4}$ and $10^{-5}$ at the very end of the path [32,35].

On the other hand a considerable amount of experimental data on cancer frequencies at low doses have been compiled in epidemiological studies during recent years [1]. This epidemiological approach considers also all the synergistic biological mechanisms involved in invivo irradiation as compared to in-vitro transformation experiments with cell cultures. If we take the cancer risk to be 10 cancers per rad per $10^{6}$ persons, as an average value for different organs and types of radiations $[1,36]$, a radiation sensitive organ of $10^{4} \mathrm{~mm}^{3}$ having $10^{6}$ cells/ $\mathrm{mm}^{3}$ of equal susceptibility, then the probability of cancer induction per cell and per rad is $10^{-15}$. If a dose of 1 $\mathrm{rad}(0.06 \mathrm{MeV} /$ cell) were delivered by $1 \mathrm{MeV}$ electrons (having a path length in tissue of about $4.3 \mathrm{~mm}$ and thus traversing about 430 cells), then about 430 electrons of 1 $\mathrm{MeV}$ initial energy must pass through a cell to deposit 1 $\mathrm{MeV}$ of energy, or at 1 rad about 25 electrons intersect the cell. Combining these numbers the probability (on average) that a single $1 \mathrm{MeV}$ electron intersecting a cell can lead to an observable tumor is about $4 \times 10^{-17}$.

Converting the data of Mole [37] for leukemia incidence in Japanese atom bomb survivors (typically exposed to doses exceeding $20 \mathrm{rad}$ ), a comparable probability that a $1 \mathrm{MeV}$ electron passing through a bone marrow cell will lead to leukemia is about $10^{-18}$.

Under the assumption [7] that the transformation cross-section equals the product of the cross-sectional area of the cell nucleus $\left(10^{-10} \mathrm{~m}^{2}\right)$ by the probability of transformation, we find that the above derived probabilities yield a mean transformation (action) cross-section for a $1 \mathrm{MeV}$ electron of about $10^{-23} \mathrm{~cm}^{2}$. This cross-section represents only a mean value, since it is averaged over the path length of an electron and neglects the fact that there is a latency period of about 10 to 20 years between the initiating energy deposition events and the manifestation of the tumor.

Transformation probabilities for alpha particles in the energy range of $1-2 \mathrm{MeV} / \mathrm{amu}$ are about $10^{-13}$ for lung cancer caused by inhaled radon decay products in man [38], and between $10^{-9}$ to $10^{-11}$ for bone cancer in man by ${ }^{239} \mathrm{Pu}$ alpha particles [39]. These numbers lead to average transformation cross-sections between $10^{-15}$ and $10^{-19} \mathrm{~cm}^{2}$. Assuming a quality factor of 20 , and allowing for differences in LET and particle range, alpha particle cross-sections show fair agreement with the electron data. However, all epidemiological in-vivo values are about $10^{12}$ times smaller than for in-vitro transformation. Since not all cells in an irradiated tissue volume are likely to be susceptible to malignant transformation, the actual probability will be higher by some orders of magnitude. Nevertheless, these numbers are still so very small compared to atomic cross-sections as to suggest contradiction. One possibility is that the linear extrapolation to low doses is invalid. An equivalent interpretation is that more than 1 electron or alpha particle must interact with the nucleus of a cell to induce malignant transformation.

Allowing for concomitant cell sterilization, the observed tumor frequency represents always the product of two probabilities, for transformation and for not being killed [40]. Having in mind that cell killing has a much higher probability than transformation, then in order to get the observed linear dose-effect relationship in the low dose region, the induction process must be a power function of dose, as the result of the cooperation of at least two energy deposition events. This has already been pointed out by Mole [37], who concludes that for leukemia incidence in Japanese bomb survivors, cancer induction is most probably a function of dose squared. For the production of osteosarcoma by alpha particles of radium in man, Marshall and Groer [41] put forward a theory which postulates two initiation events produced in a single cell by two alpha particles. Lloyd et al. [35] observed even a nearly cubic dose response for transformation of mouse embryo cells (CH 10T1/2) with 5.6 MeV alpha particles. Thus for electrons there is sufficient evidence that at least two energy deposition events (from two different electrons) in a cell are a necessary condition for carcinogenesis [40]. An equivalent interpretation, particularly for high LET radiation, might be that induction results from the transformation of two different transformed and most probably adjacent cells. However, it is important to note here that specific biological differences between different kinds of malignant disease may play an important role in their induction and consequently in their interpretation in terms of dose-effect relationships.

We wish to suggest yet a new line of evidence regarding the character of the dose-response curve which we believe has not yet been considered, namely, does one observe single or multiple tumor sites in radiation induced cancer. For doses causing an effect above the background level, many-hit processes have a steeper dose-response curve than single hit processes [12]. Hence the probability increment for many-hit processes per increment in dose may be substantially greater than for single hit processes. Since the number of cells at risk is the same, we would expect that multiple tumors are more common if the transformation leading to carcinogenesis is a many-hit process. Observations of site multiplicity have never been properly reported, but this sort of information may prove of 
value in understanding the shape of the dose-response curve at low doses.

Note that our track analyses of epidemiological data imply that cancer induction is a statistical process, like the exposure of photographic film. In both cases there is no threshold, by definition. There is only an exposure level at which the probability exceeds background. This is sometimes mistakenly identified as a threshold exposure.

Because of the competitive effect of cell killing, occurring with much higher probability, we can estimate the probability for transformation along the path of different energetic charged particles with the use of Figures 1 and 2 . There we see that heavy nuclei can only cause cancer at very high energies because all intersected cells are killed at lower energies. On the other hand, protons, deuterons, and alpha particles kill cells only at the very end of their range, being effective therefore to induce a cancer in the preceding part of their path. This allows us to estimate the probability for cancer induction from a single neutron, using information about the secondary particle spectrum in tissue $[42,43]$. There we see that for $14 \mathrm{MeV}$ neutrons, only protons, and to a lesser extent also alpha particles can transform a cell. For $1 \mathrm{MeV}$ neutrons, only protons can transform a cell (with the exception of the last cell in the path), since all heavier secondaries, e.g. $\mathrm{B}, \mathrm{C}, \mathrm{N}$, and $\mathrm{O}$ nuclei have residual ranges at which all cells are killed.

To repeat some of these conclusions for emphasis. These calculations have demonstrated that cell killing is the dominant mode of action for the intersection of high LET particles with cells. Transformation and carcinogenesis can only take place in surviving cells. A track structure interpretation of low dose effects which result from single intersections of cells therefore strongly suggests that the quality factor for radiation induced carcinogenesis should be smaller for high LET particles than for low LET radiations, practically indistinguishable from zero for heavy charged particles. This raises doubts about whether cell killing is actually an important end-point for radiation protection purposes, especially at low dose levels where only few cells are affected by heavy ions, if the cells intersected by the path of an ion can create no subsequent difficulties in later cell generations.

\section{Conclusion}

Biological effects of low doses of ionizing radiation have been reconsidered in the new and somewhat different light of the structure of particle tracks along the path of a single ion. In the calculations presented here, it is always assumed that a single cell is the sensitive site for a defined biological end-point, thus disregarding inter-cellular effects.
No particular conceptual problems are presented by 1-hit detectors, or by cell-killing with heavy ions. In these cases there is clearly an initial slope demanded by track structure theory and observed experimentally.

Questions, however, must be raised about the response of cells to electrons and other low "LET" radiations, about other biological end-points than cellular inactivation. One can only suggest that quantitative results like those found here should stimulate qualitative speculations.

When, on the average, at most only one in a hundred or one in a million $1 \mathrm{MeV}$ electrons incident on a single cell produces an observable effect in tissue, it is reasonable to ask whether indeed the action probability is not negligibly small and whether the observed effect does not arise from the cooperative effect of several electrons which interact with the nucleus of a cell. From the track structure analysis we find new bases for considering whether a low probability for a biological event is unacceptably and implausibly low, contradicting the linear extrapolation from which it was calculated. The new bases of comparison are the known cross-sections for nuclear, atomic, and molecular processes. When an action cross-section for a biological process is orders of magnitude below molecular cross-sections, when it even approaches nuclear cross-sections, we deem it to be unacceptably low. We conclude that there is an error in the logical chain which led to the inferred value of the biological cross-section. The numerical values of the crosssection we have inferred from these calculations, from the linear extrapolation to low dose, lead us to support the view that cancer induction is a "many-hit" process for electrons, and is at least a "2-hit" process for alpha particles. Especially for alpha particles we must note that to achieve two hits, that is two interactions leading to the end-point, may require more than two alpha particle passages through the nucleus of a cell. We find no basis for either a linear or a linear-quadratic extrapolation to low doses. And because of the statistical nature of these processes, we find no basis for a threshold dose.

\section{References}

[1] National Academy of Sciences Committee on Biological Effects of Ionizing Radiation, The effects on populations of exposure to low levels of ionizing radiation: 1980 (BEIR - III) (National Academy Press, Washington, D.C., 1980).

[2] B. L. Cohen, Health Phys. 39 (1980) 659.

[3] International Commission on Radiation Units and Measurements (ICRU), ICRU Report 30 (1979).

[4] M. N. Varma, H. G. Paretzke, J. W. Baum, J. T. Lyman, and J. Howard, Proc. 5th Symp. on Microdosimetry, Verbania-Pallanza, Italy, eds., J. Booz, H. G. Ebert, and B. G. R. Smith, vol. 1, Commission of the European Communities, Luxembourg 1976 (EUR 5452 d-e-f) p. 75. 
[5] M. N. Varma and J. W. Baum, Radiat. Res. 81 (1980) 355.

[6] J. Fain, M. Monnin, and M. Montret, Radiat. Res. 57 (1974) 379.

[7] R. Katz, B. Ackerson, M. Homayoonfar, and S. C. Sharma, Radiat. Res. 47 (1971) 402.

[8] J. J. Butts and R. Katz, Radiat. Res. 30 (1967) 855.

[9] R. Katz and E. J. Kobetich, Phys. Rev. 186 (1969) 344.

[10] M. Jensen and O. Mathiesen, Phys. Scripta 13 (1976) 75.

[11] R. Katz, Nucl. Instr. and Meth. 183 (1979) 257.

[12] R. Katz and F. E. Pinkerton, Nucl. Instr. and Meth. 130 (1975) 105.

[13] R. Katz, Proc. 7th Symp. on Microdosimetry, Oxford, England 1980, eds., J. Booz, H. G. Ebert, and H. D. Hartfiel, vol. 2 (Harwood Academic, 1980) p. 831.

[14] R. C. Christensen, C. A. Tobias, and W. D. Taylor, Int. J. Radiat. Biol. 22 (1972) 457.

[15] T. Alper, Cellular radiobiology, (Cambridge University Press, Cambridge, England, 1979).

[16] R. Z. Lockart, Jr., M. M. Elkind, and W. B. Moses, J. Nat. Cancer Inst. 27 (1961) 1393.

[17] R. Katz, Phys. Med. Biol. 23 (1978) 909.

[18] R. E. Meyn and H. R. Withers (eds.), Radiation biology in cancer research (Raven Press, New York, 1980).

[19] P. R. J. Burch and M. S. Chesters, Phys. Med. Biol. 24 (1979) 1216.

[20] R. Katz and S. C. Sharma, Proc. 6th L.H. Gray Conf. on Cell survival after low doses of ionizing radiations, London, ed., T. Alper, (The Institute of Physics, London, 1974) p. 78.

[21] R. A. Roth, S. C. Sharma, and R. Katz, Phys. Med. Biol. 21 (1976) 491.

[22] R. Katz and S. C. Sharma, Nucl. Instr. and Meth. 110 (1973) 93.

[23] R. Katz and S. C. Sharma, Int. J. Radiat. Biol. 26 (1974) 143.

[24] R. A. Roth and R. Katz, Radiat. Res. 83 (1980) 499.

[25] R. Katz and S. C. Sharma, Phys. Med. Biol. 21 (1976) 491.

[26] L. C. Northcliffe and R. F. Schilling, Nucl. Data Tables A7 (1970) 233.
[27] M. Schafer, R. Facius, K. Baltschukat, and H. Bucker, Proc. 7th Symp. on Microdosimetry, Oxford, England, eds., J. Booz, H. G. Ebert, and H. D. Hartfiel, vol. 2 (Harwood Academic Publishers, 1980) p. 1331.

[28] J. Kiefer, S. Rase, J. Luggen-Hölscher, E. Schneider, G. Kraft, and H. Liesem, ibid., p. 915.

[29] D. T. Goodhead, R. J. Munson, J. Thacker, and R. Cox, Int. J. Radiat. Biol. 37 (1980) 137.

[30] R. Datta, A. Cole, and S. Robinson, Radiat. Res. 65 (1976) 139.

[31] E. L. Lloyd, M. A. Gemmell, C. B. Henning, D. S. Gemmell, and B. J. Zabransky, Int. J. Radiat. Biol. 35 (1979) 23.

[32] A. Cole, R. E. Meyn, R. Chen, P. M. Corry, and W. Hittelman, Radiation biology in cancer research, eds., R. E. Meyn and H. R. Withers (Raven Press, New York, 1980) p. 33.

[33] M. M. Elkind and G. F. Whitmore, The radiobiology of cultured mammalian cells, Appendix (Gordon and Breach, New York, 1967).

[34] E. J. Hall, Biological problems in the measurement of survival at low doses, ed., T. Alper (Wiley, London, 1974) p. 13.

[35] E. L. Lloyd, M. A. Gemmell, C. B. Henning, D. S. Gemmell, and B. J. Zabransky, Int. J. Radiat. Biol. 36 (1979) 467.

[36] B. L. Cohen, Health Phys. 41 (1981) 769.

[37] R. H. Mole, Proc. 5th Int. Conf. of Radiation research, Seattle, 1974, eds., O. F. Nygaard, H. J. Adler and W. K. Sinclair (Academic Press, New York, 1975) p. 860.

[38] K. S. Parthasarathy and U. Madvanath, Proc. 2nd Special Symp. on Natural radiation environment, Bombay, 1981 (in press).

[39] A. C. James and N. F. Kember, Phys. Med. Biol. 15 (1970) 39.

[40] R. H. Mole, Brit. J. Radiol. 48 (1975) 157.

[41] J. H. Marshall and P. G. Groer, Radiat. Res. 71 (1977) 149.

[42] R. S. Caswell and J. J. Coyne, Proc. 1st Symp. on Neutron dosimetry in biology and medicine, Neuherberg/München, eds., G. Burger, H. Schraube, and H. G. Ebert, Commission of the European Communities (1972) vol. 1, p. 25.

[43] A. A. Edwards, ibid., p. 48. 